\title{
Spatio-temporal Evolvement of Rural Economic Development of Shandong
}

\author{
F. ZHAO, J.Z. ZHANG* \& Y.X. ZHU \\ College of Urban and Environment, Huaiyin Normal University, Huaian, China \\ College of Environment and Planning, Liaocheng University, Liaocheng, China \\ *Corresponding author's Email: zhangjinzong_722@163.com
}

\begin{abstract}
We assess the rural economic development level of 140 counties, districts, county-level cities of Shandong Province by using the national average value and the counties' maximum as reference values. We analyze the tendency of evolvement of rural economic and spatial status of Shandong Province. The findings are as followings: There is an obvious advantage in Shandong's rural economy compare with national average. The spatial status of rural economic is one core region lays in the east of Shandong peninsula, an development axes lays from peninsula to the middle of the Provice, there are two marginalization regions lay respectly in the Liaocheng-Heze low level region and Linyi circle low level region.
\end{abstract}

KEYWORD: 5 level regions; Rural economy; transition probability matrix; Spatio-temporal Evolvement

\section{INTRODUCTION}

Spatial structure of the regional economy refers to the various elements affecting regional economic development in the spatial distribution, the combination of status and functions of contact [1] in a particular area .From Classical to modern space structure theory and the "spatial economics" theory, we have produced a lot of theories of the evolution of regional economic spatial structure [2] According to the spatial form of economic activity, we can determine the status and function of the combination of elements, revealing the process of regional economic development and its mechanism of action, we also can provide guidance and reference for the inquiry of the factors affecting regional structure and the adjustment strategy of the regional spatial structure.

The research of Rural Economic Development in Shandong Province focused on the adjustment of rural development strategies and countermeasures, rural urbanization, rural industrial structure, influencing factors analysis of farmers' income growth, the income gap within rural residents a total of five aspects. Yang Xuecheng (1993) [3], Kong Qingliang (1994) [4], Chi Yunxiang (2000) [5] put forward development proposals for the aspects of the strategic choice from Strategies for rural development, and Shandong Agricultural development strategy. Deng Su (2006) [6], Yuan Bin (2007) [7], Li Xin (2011) [8] studied the changes in the rural economic gap in Shandong Province, the status quo of rural wealth gap and economic gap between urban and rural areas in Shandong Province, then they proposed the solving strategies.

Above studies are just studies the overall rural economy development or professional sector development in provincial-level, no studies involving spatial pattern of the rural economy. In this paper, we use the statistical analysis to calculate the per capita net income of rural residents of each county, then we compare it with the national average of per capita net income of rural resisdent and the maxima net income of each county in the same period. From the relative evaluation of the interprovince regions and the absolute evaluation comparing with the national values, we can evaluate the rural encomic development level of the entire province's county area(Municipal districts, Countylevel cities, counties).We summarize the spatial and temporal evolution characteristics of spatial pattern of rural economy on completely unit county level area in Shandong province in the year of 1999,2005 and 2012.Then we can provide theoretical support for making timely and targeted regional plannings and policy adjustments.

\section{THE PATTERN OF REGIONAL RURAL ECONOMY IN SHANDONG}

\subsection{Data and Evolution Methods}

The data comes from the Satistical Yearbook of Shandong in 2013, 2006, 2000. We use the data of per capita net income of farmers, the total population and the non-agricultural population. We select the counties per capita net income of rural residents and 
compare it with the national per capita net income of rural residents and the maximum of the counties per capita net income of rural residents. Then we calculate the location quotient of the per capita net income of rural residents in the county area of Shandong and the maximum of the per capita net income of rural residents in Shandong. After the above calculation, we can calculate the location quotients of the per capita net income of rural residents of all counties by contrast with the maximum of the per capita net income of rural residents in Shandong in the same year. We use the fifth of the lowest location quotient as the class interval. At last, we can divide the rural economic development level of Shandong in each year into five levels Type based on the location quotient. With the help of GRCGIS, we can summarize the spatial and temporal evolution characteristics of spatial pattern of Shandong Province's rural economy in the year of 1999, 2005, 2012.

\subsection{The differentiation of the type of the rural economic development level}

In this paper, we examine the differentiation of the level of Shandong Rural Economic Development from the two aspects of the differentiation of the type of the level of economic development and the spatial differentiation. According to the above five levels of type classification method, the level of rural economic development in Shandong Province' divided sections in each year as shows in Table 1:

Table 1. Rural Economic Location Quotient's interval division

\begin{tabular}{|l|c|c|c|}
\hline \multirow{2}{*}{ Level type } & \multicolumn{3}{|c|}{ Years } \\
\cline { 2 - 4 } & 1999 & 2005 & 2012 \\
\hline Minimum & 0.436 & 0.449 & 0.524 \\
\hline Interval & 0.113 & 0.11 & 0.105 \\
\hline Low level & $0.436-0.549$ & $0.449-0.551$ & $0.524-0.581$ \\
\hline Low-Middle & $0.550-0.662$ & $0.552-0.669$ & $0.582-0.685$ \\
\hline Middle level & $0.663-0.775$ & $0.670-0.780$ & $0.686-0.790$ \\
\hline Middle-High & $0.776-0.887$ & $0.781-0.890$ & $0.791-0.895$ \\
\hline High & $0.888-1$ & $0.891-1$ & $0.896-1$ \\
\hline
\end{tabular}

From Table 1, the Range of the per capita net income of rural residents in Shandong is very high. The lowest Location Quotient is 0.436. However, the development tendency of the counties' per capita net income of rural residents in Shandong is that the lowest value is gradually increased and the class interval is gradually narrowed. The lowest value has relatively increased by $20 \%$ in 13 years. The situation of the three times' development level of all counties' regional rural economic in Shandong is as follows:
Table 2. the Proportion of Rural Economic Location Quotient

\begin{tabular}{|l|c|c|c|c|c|c|}
\hline & \multicolumn{2}{|c|}{1999} & \multicolumn{2}{c|}{2005} & \multicolumn{2}{c|}{2012} \\
\hline Levels & Rurla & Total & Rurla & Total & Rurla & Total \\
\hline High level & 4.135 & 7.252 & 2.309 & 7.340 & 4.098 & 9.572 \\
\hline Middle-High level & 15.41 & 17.80 & 14.34 & 17.05 & 11.59 & 13.77 \\
\hline Middle level & 17.42 & 17.50 & 19.27 & 20.61 & 13.56 & 15.88 \\
\hline Low-Middle level & 28.4 & 27.42 & 24.78 & 22.96 & 32.23 & 29.92 \\
\hline Low-level & 34.64 & 30.03 & 39.29 & 32.08 & 38.52 & 30.86 \\
\hline
\end{tabular}

As we can see from Table 2, compared with the maximum of the per capita net income of rural residents, the per capita net income of rural residents in Shandong has small changes; From the year of 1999 to 2005, the per capita net income has increased by $2.40 \%$.From the year of 2005 to 2012 , the per capita net income of rural residents has increased by $0.63 \%$. The per capita net income of rural residents has increased by $3.04 \%$ from 1999 to 2012. In addition to the high-level group, the net income of the area's Location Quotient at all levels have a slight increase. Measured by the National Average, from 1999 to 2005, the per capita net income has increased by $6.18 \%$.From 2005 to 2012, the per capita net income of rural residents has increased by $0.70 \%$. The per capita net income of rural residents has increased by $6.92 \%$ from 1999 to 2012 .This suggests that, generally speaking, the interior relative differences in Shandong counties and cities of the per capita net income of rural residents have a slight narrow. All counties' per capita net income of rural residents are jointly improved, and this is the general trendency. With respect to the country concerned, the speed of Shandong Province's per capita net income of rural residents' improvement is slightly higher. Faster than the overall national development speed, this is the good aspect of Shandong rural economic development.

\section{CTHE SPATIAL DIFFERENTIATION OF THE LEVEL OF ECONOMIC DEVELOPMENTL}

Based on the Location Quotient of each county region's per capita net income of rural residents in the year of 1999 and 2012, according to the aforementioned criteria for the five classification, we can get the map of the level of regional economic development in the counties of Shandong. The main consideration is that the individual enclave area surrounded by a certain type of area will be classified as a type of zone around. Meanwhile, compared with the regional agglomeration type and the spatial neighborhood relation, under the principles of spatial intergrity and zonation intergrity, we will classify the region of which the individual levels are close to a type of a regional agglomeration which is adjacent to the region into 
this type. The specific standard is that the relative ratio of the county or the city's score and that type's standard scores is higher than $88 \%$.According to this, we can get the patterns of the rural economic development levels in Shandong's county regions for two years.

The zonation of the Province's rural economic level in 1999 can be divided into: high-level Peninsula region, mid-level and high-level peninsula region, Jinan-Zibo high-level and mid-level region, medium level peninsula region, Jinan-Zibo medium level region, Jining medium level region, North Shandong mid-level and low-level region, central south of Shandong mid-level and low-level region, southeast of Shandong mid-level and low-level region, North Shandong low-level region, Liaocheng-Heze low-level region, Yimeng low-level region, a total of 5 levels and 12 regions.

High-level peninsula region includes 3 parts, 12 counties. They are Qingdao, Weihai, Yantai highlevel regions. The high-level regions sporadically distribute in the peninsula. Its maximum value of the Location Quotient is 0.916. The rural population accounts for $4.30 \%$ of the total population in Shandong Province, and the region population accounts for $5.42 \%$ of the total population in Shandong Province. The high-level and mid-level region includes 2 parts, 27 counties. They are midlevel and high-level peninsula region, Jinan-Zibo high-level and mid-level region. Its maximum value of the Location Quotient is 0.823. The rural population accounts for $16.12 \%$ of the total population in Shandong Province, and the region population accounts for $19.42 \%$ of the total population in Shandong Province. The medium level region includes 3 parts, 25 counties. They are medium level peninsula region, Jinan-Zibo medium level region, Jining medium-level region. Its maximum value of the Location Quotient is 0.717. The rural population accounts for $18.91 \%$ of the total population in Shandong Province, and the region population accounts for $18.90 \%$ of the total population in Shandong Province. The mid-level and low-level region includes 3 parts, 39 counties. They are north Shandong mid-level and low-level region, central south of Shandong mid-level and low-level region, southeast of Shandong mid-level and lowlevel region. Its maximum value of the Location Quotient is 0.605. The rural population accounts for $28.57 \%$ of the total population in Shandong Province, and the region population accounts for $28.28 \%$ of the total population in Shandong Province. The low-level region includes 3 parts, 36 counties. They are North Shandong low-level region, Liaocheng-Heze low-level region, Yimeng low-level region. Its maximum value of the Location Quotient is 0.501 . The rural population accounts for $32.09 \%$ of the taotal population in Shandong Province, and the region population accounts for $27.97 \%$ of the total population in Shandong Province.
The zonation of the Province's rural economic level in 2012 cinsist of 5 levels and 9 regions, as shown in Figure 2. The high-level includes 3 parts: Qingdao high-level region, Weihai high-level region, Yantai high-level region. This high-level region consists of 16 county areas. Its maximum value of the Location Quotient is 0.925. The rural population accounts for $3.82 \%$ of the total population in Shandong Province, and the region population accounts for $6.71 \%$ of the total population in Shandong Province. The high-level and mid-level region includes 2 parts, 25 counties. Its maximum value of the Location Quotient is 0.819. The rural population accounts for $14.38 \%$ of the total population in Shandong Province, and the region population accounts for $17.99 \%$ of the total population in Shandong Province. The mid-level region has evolved into a large area from the peninsula and estuary to the central south Shandong Province. It includes 21 counties and cities. Its maximum value of the Location Quotient is 0.728 . The rural population accounts for $10.34 \%$ of the total population in Shandong Province, and the region population accounts for $13.18 \%$ of the total population in Shandong Province. The mid-level and low-level region includes North Shandong mid-level and low-level region, central south of Shandong mid-level and low-level region, southeast of Shandong mid-level and low-level region, a total of 3 parts. The north Shandong mid-level and low-level region consists of 23 counties and cities, and the central south of Shandong mid-level and low-level region has 17 counties and cities. The southeast of Shandong mid-level and low-level region has 6 counties and cities. Its maximum value of the Location Quotient is 0.625 . The rural population accounts for $34.55 \%$ of the total population in Shandong Province, and the region population accounts for $31.24 \%$ of the total population in Shandong Province. The low-level region has evolved into 2 portions. They are Liaocheng-Heze low-level region with 18 counties and cities, and Yimeng low-level region with 15 counties and cities. The maximum value of the Location Quotient of the low-level region is 0.542 . The rural population accounts for $36.91 \%$ of the total population in Shandong Province, and the region population accounts for $30.89 \%$ of the total population in Shandong Province.

From the comparison of the patterns of the above two graphs, we can clearly see the evolution of Shandong Province Rural Economy. The economic structure of 1999 is that the core area relatively concentrates in the region from peninsula to JinanZibo area. Low-level region concentrates in Liaocheng-Heze area, Yimeng area and north Shandong. Adjacent to the high-level region and mid-level and high-level region is the medium level peninsula region and Jinan-Zibo medium level region. These mid-level and low-level regions are 
embedded in the areas between the low-level regions and the medium levels of central Shandong and central south Shandong. The economic structure of 2012 still is the core area concentrates in the region from peninsula to Jinan-Zibo area. Its importance has been further strengthened. The low-level region concentrates in Liaocheng-Heze low-level region, Yimeng low-level region. Jinan-Zibo mid-level and high-level regions are embedded in the mid-level region of peninsula, estuary and central Shandong. Mid-level and low-level regions are embedded in the low-level regions and peninsula, estuary and the medium level regions of central Shandong.

Two years' framework of the pattern of Shandong Economic spatial differentation is very similar, and the peninsula-inland differentiation is obvious. The third change is that a small pieces of the low-level region, which belongs to the north Shandong, has evolved into the mid-level and high-level region. The fourth change is that Linyi low-level region has extended to south. The fifth change is that Jining medium level region is joining into the mid-level and low-level region of south Shandong. Until 2012, the spatial pattern of shandong rural economy is that the high-level region and mid-level and high-level region of peninsula forms a core. The medium level from peninsula, estuary to central Shandong and the Zibo-Jinan mid-level and high-level region forms a development axis. Western Liaocheng-Heze lowlevel region and southeast of Yimeng low-level region is the "Two marginalized areas". The north Shandong which runs through north and south and the central south Shandong is the "Two slight backward areas".

According to the changes of the county economic's Location Quotient of these three years, the rural economic's focal risen in Shandong Province should be the central and east area of peninsula. The estuary area is the secondary focal risen area. Liaocheng-Heze low-level region and Yimeng low-level region is a steadily suatained lowlevel. The overall pattern of the level of Shandong rural economy will not change in a long period of time.

\section{CONCLUSION}

Compared with the national average, the rural economy of Shandong Province is rapidly to a good momentum. Because of a greater advantage of Shandong Province Rural Economic compared with National Rural Economic, from the distribution of the population, we can see a skewed distribution of which the low-level has a high portion, the highlevel has a low persentage steply. From this perspective, the current development of Shandong Province Rural Economic is unsatisfactory. It is still in the early and middle days before reaching in the advanced stage. The level of per capita net income of rural residents in Shandong is much better than the level of GDP of the county area. Shandong has the advantage of a relatively better development of rural economic. From the distribution of the total population, we can see that the high-level and lowlevel region of the Rural Economic Development in Shandong showed a characteristic of a slight polarization, meanwhile, the populations and the number of the low-level region increases faster than high-level region.

The space differentiation pattern of peninsula inland differentiation is very obvious in Shandong province rural economic. Until 2012, Shandong rural economic has formed a spatial pattern of "a core," "a development axis", "two marginalized regions," "two slight backward areas". It can be predicted that, the rural economic's focal risen in Shandong Province should be the central and east area of peninsula. The estuary area is the secondary focal risen area. Liaocheng-Heze low-level region and Yimeng low-level region is a steadily suatained lowlevel.

\section{ACKNOWLEDGMENT}

This research has funded by the social science project from department of science and technology of Shandong Province, (No.41271135), we express our thanks to the office-holders. The authors would also like to express appreciations to colleagues in our laboratory for their valuable comments and other helps.

\section{REFERENCES}

[1] Zhang.J.Z\&Zhu.YX. The Spatiotemporal pattern of economic evolution in Xinjiang Counties. Arid Rrea resources and Environments, 2013(2), pp.24-29

[2] Friedmann.J.R. 1966.Regional development policy: a case study of Venzuela. Cambridge, Mas: MIT Press

[3] Yang.XC. The Gradient Propulsion and the Leaping Development. Agricultural Economic Question,1993 (9), pp.93-96

[4] Kong.QL. Development Strategy Research of Shandong. Shandong Social Science, 1994 (4), pp.93-97

[5] Chi.Y.X. Agricultural Sustainable Development Strategy of Shandong. Human Geography, 2000. (2), pp.106-109

[6] Deng.S. \& Zhang.X. The change trend of rural economic gap and inner composition in Shandong. Dongyue Tribune, 2006(4), pp.84-89

[7] Yuan.B. The empirical analysisof the root cause of rural economic gap in Shandong. Northern Economy, 2007(10), pp.63-68

[8] Li.X. Guo.N. \& Sun Y.W. Urban-rural economic differences status quo and the countermeasures of coordinated development. Guizhou Agricultural Sciences, 2012(4), pp.31-36 